\title{
The effect of NFATc1 on vascular generation and the possible underlying mechanism in epithelial ovarian carcinoma
}

\author{
LONG LI ${ }^{1}$, JIHUI YU ${ }^{1}$, ZHAONING DUAN ${ }^{2}$ and HONG-XING DANG ${ }^{3-5}$ \\ Departments of ${ }^{1}$ Physical Examination and ${ }^{2}$ Gynecology, The First Affiliated Hospital of Chongqing Medical University; \\ ${ }^{3}$ Department of PICU, Children's Hospital of Chongqing Medical University, Ministry of Education Key Laboratory of \\ Child Development and Disorders, ${ }^{4}$ China International Science and Technology Cooperation base of Child Development \\ and Critical Disorders ${ }^{5}$ Chongqing Engineering Research Center of Stem Cell Therapy, Chongqing 400016, P.R. China
}

Received November 6, 2015; Accepted December 16, 2015

DOI: $10.3892 /$ ijo.2016.3355

\begin{abstract}
We investigated the effect of nuclear factor of activated T cells c1 (NFATc1) on the growth and vascular generation of human ovarian carcinoma SKOV3 celltransplanted tumors in nude mice and explored the possible underlying mechanism. NFATc1 siRNA was transfected into the SKOV3 cells, which were then subjected to immunofluorescence tests and real-time reverse transcription polymerase chain reaction (RT-PCR) to determine the transfectioninduced inhibition rate. The tumor volumes in the nude mice in all groups were measured to determine the in vivo antitumor effect of NFATc1 siRNA. Immunohistochemical (IHC) methods were employed to detect NFATc1 expression in tumor tissue, combined with cytokeratin $(\mathrm{CK})$ staining to label the epithelial origin of the tumor tissue. CD34 and podoplanin were used as markers for labeling microvessels and microlymphatic vessels, respectively. The densities of microvessels and microlymphatic vessels in each group were calculated and statistically analyzed. RT-PCR and western blotting were performed to detect the protein and mRNA expression levels of NFATc1, the ELR ${ }^{+}$CXC chemokine interleukin (IL)-8, fibroblast growth factor-2 (FGF-2), and platelet-derived growth factor BB (PDGF BB) in xenografted tumor tissue in all groups. NFATc1 was highly expressed in tumor tissue in the control groups. The intervention group exhibited a tumor growth inhibition rate of $57.08 \%$ and presented a lower tumor weight and volume compared with the two control groups. In the control groups, the microvessel densities were $12.00 \pm 1.65$ and $11.47 \pm 0.32$, respectively, and the microlymphatic vessel densities were $10.03 \pm 0.96$ and $9.95 \pm 1.12$; these values were significantly higher than in the intervention group. RT-PCR and western blot shows that NFATc1 siRNA could markedly
\end{abstract}

Correspondence to: Dr Hong-Xing Dang, Children's Hospital of Chongqing Medical University, Chongqing 400016, P.R. China E-mail: docdang@163.com

Key words: nuclear factor of activated $\mathrm{T}$ cells c1, ovarian cancer, vascular generation, aggressiveness suppress the expression of IL-8, FGF-2 and PDGF BB at the mRNA and the protein level. In conclusion, it was shown that NFATc1 siRNA significantly suppresses the growth and vascular generation of SKOV3 human ovarian carcinoma celltransplanted tumors subcutaneously xenografted into nude mice. The downregulation of the expression of IL-8, FGF-2 and PDGF BB may be one of the mechanisms underlying the above inhibitory effects.

\section{Introduction}

Nuclear factor of activated T cells (NFAT) c1 is an NFAT family member. NFATs are unique signal transcription factors in vertebrates and play pivotal roles in a variety of cellular functions. NFATs induce various important cellular biological processes, including the generation and activation of lymphocytes and differentiation of myocardial cells $(1,2)$. The classical pathway involved in the functional activity of NFAT was first described in the lymphocytes. Activated NFAT is released from the endoplasmic reticulum system into the cytoplasm in a pattern similar to the flow of $\mathrm{Ca}^{2+}$ through membrane $\mathrm{Ca}^{2+}$ channels activated by an extracellular stimulus. Subsequently, NFAT in the cytoplasm is highly phosphorylated. When the cell releases the $\mathrm{Ca}^{2+}$ flow, triggered by a stimulus, NFAT is dephosphorylated by calcineurin and translocates into the nucleus, promoting gene transcription through synergistic effects with other factors and activators. NFAT interacts with its DNA targets in various forms. It can bind to DNA in the form of single or heteromorphic spliceosomes, and the binding site strongly attracts other transcription factors (3). An ideal example is that activator protein (AP-1) (including Fos-Jun) binds to NFAT and DNA to form a complex, generating a necessary initial transcription element for the activation of $\mathrm{T}$ cells $(4,5)$. Moreover, NFATs are involved in cellular activities and differentiation processes in combination with other transcription factors, such as the zinc-finger transcription factor GATA-4, early growth response gene (EGR), myocyte enhancer factor-2 (MEF2), and forkhead box P3 (Foxp3), which plays an extremely important role in malignant tumors (6).

The initial research on NFATs involved exploration of their function in T cells. As an activatable nuclear factor, NFAT binds to the interleukin (IL)-2 promoter during the activation 
process of T cells (7). NFAT is also an important contributor in immunotherapy. Cyclosporine A can specifically downregulate the classic NFAT signaling pathway and has therefore been widely used to prevent the rejection of transplanted organs in clinical settings. Two decades of research has gradually revealed that NFAT transcription factors are expressed not only in the lymphocytes but also in the cells of other types of tissue, including epithelial cells. This response explains the novel finding in a number of recent key studies that NFATs have a very important function: they are closely associated with the occurrence and progression of malignant tumors. Each subtype of NFAT has a distinct function. The tumorigenic activity of NFATc1 has been confirmed (8). Previous studies found that the transformed phenotypes of NFATc1-induced fibroblast NIH 3T3 cells are readily evident, indicating that NFATc1 can sufficiently induce NIH 3 T3 cells to produce definitive transformation markers and thus verifying that NFATc1 is a potential oncogene (9). As mentioned above, calcineurin is a major regulator of NFATc1, and calcineurin activity is a necessary basis for malignant tumor progression, suggesting a close relationship between NFATc1 and tumorigenesis (10). In addition, studies have demonstrated that NFATc1 expression is associated with other types of malignant tumors; e.g., NFATc1 is a necessary key factor involved in the occurrence and progression of colon and pancreatic carcinomas $(11,12)$.

Tumor angiogenesis is a key factor deciding the occurrence and progression of solid human tumors. It can cause proliferation of tumor cells and accelerate malignant behavior such as tumor invasion, migration, and dissemination $(13,14)$. In addition, studies in recent years have shown that lymphangiogenesis plays a similar role to angiogenesis in the occurrence and progression of malignant epithelial tumors and that it even occurs prior to angiogenesis in these tumors at a very early stage. Therefore, exploration of angiogenesis and lymphangiogenesis in malignant tumors is of great significance for clarifying the mechanisms underlying disease occurrence and progression and for identifying effective treatment target genes. NFATc1 is a key factor involved in cardiovascular development during embryo formation $(15,16)$. After birth, it can still regulate the growth, differentiation, and life cycle of endothelial cells. An increasing amount of data have demonstrated that NFATc1 regulates vasculogenic responses $(17,18)$. A study using vascular endothelial growth factor (VEGF) to induce angiogenesis revealed that NFATc1 is a key component distributed at the merge point of the VEGF-induced vasculogenic endothelial cell escape pathway. NFATc1 is pivotal in the induction process of the majority of genes by VEGF/IL-1. Within the upstream portion of the NFATc1 signaling pathway, VEGF-A not only is a key factor in tumor angiogenesis but also facilitates tumor lymphangiogenesis (19). In addition, combined with EGR-1, NFATc1 can activate the tissue factor (TF, transcription of tissue factor) gene (20). The TF gene is an initiator of blood coagulation and angiogenesis. At the site of TF initiation, NFATc1 binds tightly to NF- $\kappa$ B at the overlap point, indicating that the complete transcription reaction of VEGF-A for TF or other potential genes requires the involvement of NFATc1. NFAHc1 also plays an important role in lymphangiogenesis. Its expression has been detected in developing and mature lymphatic vessels. Suppressing NFAHcl activity by controlling the expression of podoplanin and vascular endothelial growth factor receptor-3 (VEGFR-3) in lymphatic endothelial cells leads to a reduction of VEGFA-induced lymphangiogenesis in lung injury (21). Previous studies have shown that NFATc1 can act as a downstream signaling molecule of VEGF-C to interact with pro-lymphangiogenesis factors such as Prox1, podoplanin, Foxc2 and VEGFR-3, thus affecting lymphangiogenesis, particular in terms of spatial construction and channel shaping (22). All of the above studies demonstrated the important role and functions of NFATc1 in angiogenesis and lymphangiogenesis. However, studies on the role of NFATc1 in tumor vascular generation are scarce and have mainly focused on its upstream gene VEGF. To the best of our knowledge, no related studies on epithelial ovarian carcinoma have been reported. Thus, in this study we explored the effect of NFATc1 on angiogenesis and lymphangiogenesis in epithelial ovarian carcinoma in vivo and in vitro as well as the relationships of NFATc1 with ELR+ CXC chemokine IL-8, fibroblast growth factor-2 (FGF-2), and platelet-derived growth factor BB (PDGF BB) (these three factors independently play important roles in tumor angiogenesis and lymphangiogenesis). This study aimed to provide a further explanation of the effect of NFATc1 on the malignant behavior of epithelial ovarian carcinomas and to reveal the molecular mechanisms underlying NFATc1 signal transduction and the occurrence and progression of epithelial ovarian carcinoma, to gain additional knowledge to provide a theoretical basis for further research on tumor angiogenesis and lymphangiogenesis.

\section{Materials and methods}

The SKOV3 ovarian cancer cell line was from Molecular Medicine and Cancer Center, Chongqing Medical University, and the 4- to 8-week-old BALB/c athymic female nude mice from the Animal Experimental Center, Chongqing Medical University.

The SKOV3 ovarian cancer cell line was studied in the following groups: blank group; negative control group; small interfering RNA (siRNA)1, siRNA-880; siRNA2, siRNA1169; siRNA3, siRNA-1307.

The $18 \mathrm{BALB} / \mathrm{c}$ athymic female nude mice, 4 - to 8-weekold, were devided into 3 groups: blank group, mice were given injections of SKOV3 cells without any interference. Negative control group, mice were given injections of SKOV3 cells that expressed other unrelated DNA oligos. siRNA group, mice were given injections of SKOV3 cells that expressed NFATc1 siRNA with the highest transfection efficiency.

NFATc1 rabbit polyclonal antibody (ab25916); CK (BM0030); CD34 (SC-9095); IL-8 rabbit polyclonal antibody (CSB-PA08327A0Rb); FGF-2 monoclonal antibodies (sc-365106); PDGF BB rabbit polyclonal antibody (sc-7878) were used.

Cell culture and NFATc1 siRNA. The SKOV3 ovarian cancer cells were maintained in media containing $10 \%$ fetal bovine serum, $2 \mathrm{mmol} / \mathrm{l} \mathrm{L}$-glutamine, penicillin $(100 \mathrm{U} / \mathrm{ml})$, and streptomycin $(100 \mu \mathrm{g} / \mathrm{ml}$ ). Three DNA oligos (siRNA-880 antisense, 5'-UUCCGGCACAGUCAAUGACGGCUCG-3' and sense, 5'-CGAGCCGUCAUUGACUGUGCCGGAA-3'; siRNA-1169 antisense, 5'-AGAGAAUUCGGCUUGCACAGGUCCC-3' 
and sense, 5'-GGGACCUGUGCAAGCCGAAUUCUCU-3'; and siRNA-1307 antisense, 5'-AGACGUAGAAA CUGACGU GAACGGG-3' and sense, 5'-CCCGUUCACGUCAGUUU CUACGUCU-3') were designed as green fluorescent siRNA against NFATc1 mRNA to target the open reading frame of NFATc1 cDNA. These DNA oligos were contained in Lipofectamine $^{\mathrm{TM}} 2000$ and were used to infect SKOV3 cells; the highest transfection efficiency oligo was selected by RT-PCR to use for interference.

Tumor formation in nude mice. To evaluate the ability of cells to form tumors, 4- to 8-week-old BALB/c athymic female nude mice were given bilateral injections of SKOV3 tumor cells, with a total of 18 mice used. All mouse experiments were performed in accordance with institutional guidelines approved by the Institutional Animal Care and Use Committee. Each subcutaneous injection consisted of $5 \times 10^{6}$ SKOV3 cells $(0.2 \mathrm{ml})$. Control mice were injected with SKOV3 cells that expressed NFATc1 siRNA. The mice were kept in a specific pathogen-free environment and were checked every 2 days for 30 days. After 30 days, the mice were sacrificed by exposure to $5 \%$ carbon monoxide. The tumor inhibition rate was calculated with the use of the tumor weight: tumor inhibition rate $(\%)=($ control weight-experimental weight $) /$ control weight $\mathrm{x} 100 \%$. The tumor volume was calculated with the use of the following formula: tumor volume $\left(\right.$ in $\left.\mathrm{mm}^{3}\right)=1-2 \mathrm{x} \mathrm{LW}$ (L, length; $\mathrm{W}$, weight). All tumors for each group were excised, fixed in 10\% formalin overnight, and subjected to routine histologic examination and immunostaining of NFATc1/CK/CD34 by investigators who were blinded to the tumor status. The assay was repeated twice. RT-PCR, and western blot analysis were performed to observe IL-8/FGF-2/PDGF BB gene and protein expression.

Immunohistochemical (IHC) staining and analysis. IHC staining for NFATc1 and cytokeratin were performed using avidin-biotin-peroxidase methods. Briefly, tissue slides were deparaffinized in xylene and rehydrated in a graded series of ethanol, and the sections were subjected to antigen retrieval by boiling in $0.01 \mathrm{~mol} / \mathrm{l}$ sodium citrate buffer $(\mathrm{pH} 6.0)$ in a microwave oven for $10 \mathrm{~min}$. After blocking endogenous peroxidase activity with $0.3 \%$ hydrogen peroxide and blocking nonspecific protein binding with $1.5 \%$ normal goat serum, the sections were incubated overnight with an antibody at $4^{\circ} \mathrm{C}$ in a humidified chamber. Then, the sections were incubated with biotinylated goat anti-mouse $\mathrm{IgG}$ for $30 \mathrm{~min}$ and detected with the LSAB system (Dako). Sections were lightly counterstained with hematoxylin. The primary antibody was replaced with 1X PBS as a negative control. Entire tissue sections were evaluated by HScore values, which are objective measurements of staining intensity, and the percentage of tumor cells that stained positive. Five fields of each slice were randomly selected with a magnification of $x 400$, and the numbers of positive cells were counted per 100 cells/field. Staining intensity was scored as negative ( $<5 \%$ of positive tumor cells), $1^{+}$(mild intensity), $2^{+}$(moderate intensity), or $3^{+}$(intensity greater than that of the positive control). The sections were evaluated by two pathologists with a double-blind method to determine the immunohistochemistry results. The formula was HScore $=3 \mathrm{pi}$ $(i+1)$, where $\mathrm{i}$ indicates the intensity of staining, $\mathrm{Pi}$ is the percentage of cells with positive staining/the total number of tested cells, and 1 is the correction factor.

The blood vascular endothelium and lymphatic vascular endothelium were labeled using CD34 and podoplanin as markers, respectively, and then observed microscopically to determine the numbers of positively stained endothelial cells in the blood and lymphatic vessels and the morphology of microvessels and microlymphatic vessels under an optical microscope. Counting of vessels was conducted according to the following procedures: first, the areas with a high density of stained cells in each section were identified under a low magnification (x40). Next, under a high magnification (x200 or $\mathrm{x} 400$ ), the positively stained lymphatic vessels were identified and counted. The density of microlymphatic vessels in each section was obtained by dividing the mean number of microlymphatic vessels in five high-magnification view fields (x200 or x400) by the field area. Each section was read by two pathologists using a double-blind method. The number of blood microvessels was counted following procedures similar to those described above.

RNA isolation and reverse transcriptase polymerase chain reaction. RNA was isolated from SKOV3 cells (treated by siRNA and control) using RNeasy mini kits (Qiagen, Santa Clarita, CA, USA) according to the manufacturer's instructions. The RNA was eluted with water, stored at $-70^{\circ} \mathrm{C}$ and evaluated by agarose electrophoresis. For complementary DNA (cDNA) synthesis, $\sim 1 \mu \mathrm{g}$ of total RNA was transcribed with cDNA transcription reagents (PE Biosystems, Foster City, CA, USA) using random hexamers, according to the following conditions: $30^{\circ} \mathrm{C}$ for $10 \mathrm{~min} ; 50^{\circ} \mathrm{C}$ for $20 \mathrm{~min} ; 99^{\circ} \mathrm{C}$ for $5 \mathrm{~min}$; and $5^{\circ} \mathrm{C}$ for $5 \mathrm{~min}$.

The PCR conditions were as follows: $\beta$-actin, $95^{\circ} \mathrm{C}, 5 \mathrm{~min}$, 1 cycle; 95 and $59.5^{\circ} \mathrm{C}, 30 \mathrm{sec}, 30$ cycle; $72^{\circ} \mathrm{C}, 30 \mathrm{sec} ; 72^{\circ} \mathrm{C}$, $10 \mathrm{~min}, 1$ cycle. NFATc 1 gene, $95^{\circ} \mathrm{C}, 5 \mathrm{~min}, 1$ cycle; 95 and $56^{\circ} \mathrm{C}, 30 \mathrm{sec}, 30$ cycle; $72^{\circ} \mathrm{C}, 30 \mathrm{sec} ; 72^{\circ} \mathrm{C}, 10 \mathrm{~min}, 1$ cycle. CXCR2 gene, $95^{\circ} \mathrm{C}, 5 \mathrm{~min}, 1 \mathrm{cycle} ; 95$ and $57^{\circ} \mathrm{C}, 30 \mathrm{sec}$, 30 cycle; $72^{\circ} \mathrm{C}, 30 \mathrm{sec} ; 72^{\circ} \mathrm{C}, 10 \mathrm{~min}, 1$ cycle. FGF- 2 gene, $95^{\circ} \mathrm{C}$, 5 min, 1 cycle; 95 and $58^{\circ} \mathrm{C}, 30 \mathrm{sec}, 30$ cycle; $72^{\circ} \mathrm{C}, 30 \mathrm{sec}$; $72^{\circ} \mathrm{C}, 10$ min, 1 cycle. PDGF BB gene, $95^{\circ} \mathrm{C}, 5$ min, 1 cycle; 95 and $58^{\circ} \mathrm{C}, 30 \mathrm{sec}, 30 \mathrm{cycle} ; 72^{\circ} \mathrm{C}, 30 \mathrm{sec} ; 72^{\circ} \mathrm{C}, 10 \mathrm{~min}, 1$ cycle.

The PCR primer sequences were as follows: $\beta$-actin [546 base pair (bp)], 5'-CTCGTCATACTCCTGCTTGCT-3' and 5'-CGGGACCTGACTGACTACCTC-3'. NFATc1 (381 bp), 5'-CGATCCCGGGGTAGCAGCCT-3 and 5'-CACCGCCATA CTGGAGCCGC-3. IL-8 (222 bp), 5'-GCCCTGACAGCTCC CAAGCCT-3' and 5'-ATGCGTCATGCCGCTTCCCAG-3'. FGF-2 (298 bp), 5'-CAGTGAGTGCCGACCCGCTC-3' and 5'-GCGGGAAGACAGCCAGTCCG-3'. PDGF BB (104 bp), 5'-CCCTGCTCCACAAAGGCGGG-3' and 5'-CCTAGCCCG GTGCCTCGTCT-3'.

Polymerase chain reaction products were visualized by ethidium bromide staining after $2 \%$ agarose gel electrophoresis. Following PCR, $5 \mu \mathrm{l}$ of the gene amplification product was subjected to $1.5 \%$ agarose gel electrophoresis. The grayscale values of the band sizes for NFATc1, IL-8, FGF-2, PDGF BB, and the internal reference $\beta$-actin were analyzed using a Quantity One Gel Imaging system. The optical density (OD) ratios of NFATc1/ $/ \beta$-actin, IL- $8 / \beta$-actin, FGF- $2 / \beta$-actin, and PDGF BB/ $\beta$ actin were calculated and compared among the intervention group, the negative control group, and the blank control group. 
Western blot analysis. Total protein extracts from SKOV3 cells and transplanted tumor tissue were obtained using analysis buffer, and equal amounts ( $30 \mu \mathrm{g} / \mathrm{load})$ were analyzed by immunoblotting. The antibody against $\beta$-actin was obtained from Sigma-Aldrich (A5441, 1:20,000). Antibodies against NFATc1 (ab25916, 1:1,000); against IL-8 (CSB-PA08327A0Rb):1:800; FGF-2 (sc-365106):1:500; and PDGF BB (sc-7878):1:800. The secondary antibodies were anti-rabbit immunoglobulin horseradish peroxidase-linked $\mathrm{F}(\mathrm{ab}) 2$ fragment from donkey (Amersham Biosciences). Western blot reagents were from an electrochemiluminescence kit (Amersham Biosciences).

Determination of protein concentrations: first, a $100 \mathrm{mg} / \mathrm{ml}$ bovine serum albumin (BSA) solution and a Coomassie brilliant blue G250 solution were prepared, and the standard protein sample was fully dissolved in the solution. Next, $10 \mu \mathrm{l}$ of the protein solution was diluted to $100 \mu 1$ to reach a desired final concentration of $0.5 \mathrm{mg} / \mathrm{ml}$. Subsequently, $0,1,2,4,8,12$, 16 and $20 \mu \mathrm{l}$ of the standard protein solution was added to the wells of a 96-well plate, and each well was brought to a final volume of $20 \mu \mathrm{l}$ by adding more of the solution used for dilution. Appropriate amounts of the samples to be tested were then added to the sample wells of the 96-well plate and brought to a final volume of $20 \mu \mathrm{l}$ with the same solution used for dilution. Finally, $200 \mu \mathrm{l}$ of the working solution for the bicinchoninic acid (BCA) assay was added to all wells after being left to stand at $37^{\circ} \mathrm{C}$ for $30 \mathrm{~min}$. OD values at a wavelength of $562 \mathrm{~nm}$ (A562) (a wavelength range of 540-595 $\mathrm{nm}$ was considered acceptable) were detected. The protein concentration was determined based on the standard curve.

Semi-quantitative analysis of the bands: the bands obtained after electrophoresis were imaged using a ChemiDocXRS chemiluminescence imaging system and analyzed using Quantity One 4.5.2 software. The areas and grayscale values of the bands were semi-quantitated based on those of the first lane as a reference and then compared.

Statistical analysis. Measurement data were expressed as the mean \pm standard deviation. Comparison of mean values among multiple samples was conducted through analysis of variance (ANOVA), and multiple comparisons among multiple samples were performed using Student-Newman-Keuls (SNK)-q tests. All statistical analyses were carried out with SPSS 16.0 software, and a P-value of $<0.05$ indicated that the difference was statistically significant.

\section{Results}

Transfection efficiency. The transfection efficiency of each group was determined after 24 and $48 \mathrm{~h}$ of transfection using a fluorescence microscope. Green fluorescent particles appeared in the SKOV3 cells in all three groups, suggesting that siRNA had been successfully transfected into the cells. The transfection rate at $48 \mathrm{~h}$ after transfection was $59.1 \%$ for group A, $85.3 \%$ for group B, and $51.9 \%$ for group C, which was higher than the rates at $24 \mathrm{~h}$ after transfection in all groups. Group B showed the highest transfection efficiency at $48 \mathrm{~h}$ post-transfection, presenting significant differences compared with the other two groups (Fig. 1).

RT-PCR was used to determine the suppression level of NFATc1 gene expression by siRNA in each group at
$48 \mathrm{~h}$ post-transfection. The results showed that the NFATc1 gene expression levels in the cells of the three groups were $0.532 \pm 0.001,0.278 \pm 0.001$ and $0.498 \pm 0.003$, respectively. Group B presented the highest inhibition rate at $48 \mathrm{~h}$ posttransfection, showing significant differences from the rates of the other two groups $(\mathrm{P}<0.01)$, while no significant difference was observed between group A and C (Fig. 2 and Table I).

Based on comparison of the results for the transfection efficiency and inhibition rate among the three siRNA sequences, siRNA-1169, which was used in group B and showed the highest transfection efficiency, was chosen for subsequent experiments. Three groups were set up in the subsequent experiments: A, the blank group; B, the negative control group; and $\mathrm{C}$, the intervention group.

Suppression of NFATcl activity results in growth inhibition of xenografted tumors. To investigate whether NFATc1 downregulation is associated with in vitro and in vivo tumor growth in ovarian cancer cells, we investigated mouse xenograft tumor growth after inoculation. The success rate of tumor xenografting in 18 nude mice reached $100 \%$, with latent periods of $\sim 6-8$ days in the blank control group and the negative control group and $\sim 8-11$ days in the NFATc1 siRNA intervention group. The tumor grew expansively at the xenografting site, and its volume gradually increased after 2-3 weeks. All of the tumor-bearing nude mice survived until the end of experiments. Observations performed with the naked eye revealed a smaller tumor size in the NFATc1 siRNA intervention group compared with the other control groups. The mean weight, volume, and inhibition rate of tumor growth in the three groups are presented in the Table II. The NFATc1 siRNA intervention group exhibited a growth inhibition rate of $57.08 \%$ and a lower weight and volume than the two control groups; these differences were significant (Table II).

NFATcl is highly expressed in the xenograft tumor tissue. We examined NFATc1 expression in xenograft tumor tissue by IHC staining. As shown in Fig. 3 and Table III, control group expressed high levels of NFATc1 compared with siRNA group. Of the 30 samples/group. The mean NFATc1 HScore for control groups were 320 (range, 0-356), 336 (range, 0-356); the HScore of the siRNA samples was 35 (range, 0-180). This finding was statistically significant $(\mathrm{P}<0.05)$.

Suppressing NFATcl activity leads to inhibition of angiogenesis and lymphangiogenesis in epithelial ovarian carcinoma tumor tissue in a xenograft nude mouse tumor model. To confirm whether NFATc1 is associated with tumor angiogenesis and lymphangiogenesis, we first examined the xenograft mouse tumor tissues generated from animals injected with SKOV3/NFATc1 siRNA-1169. The overall blood vessel density and lymph vessels were decreased statistically in tissues expressing NFATc1 siRNA, as indicated by the reduced number of tissue microvessels stained with CD34 and podoplanin (Fig. 4 and Table III). The brown-yellowish-stained cytokeratin (CK) antigen appeared specifically in the cytoplasm of epithelial cells in all tissue sections of the xenografted tumors, confirming the epithelial origin of the xenografted tumors. CD34 staining using an anti-CD34 antibody revealed the angiogenesis status in the tumor tissue of each group: CD34 


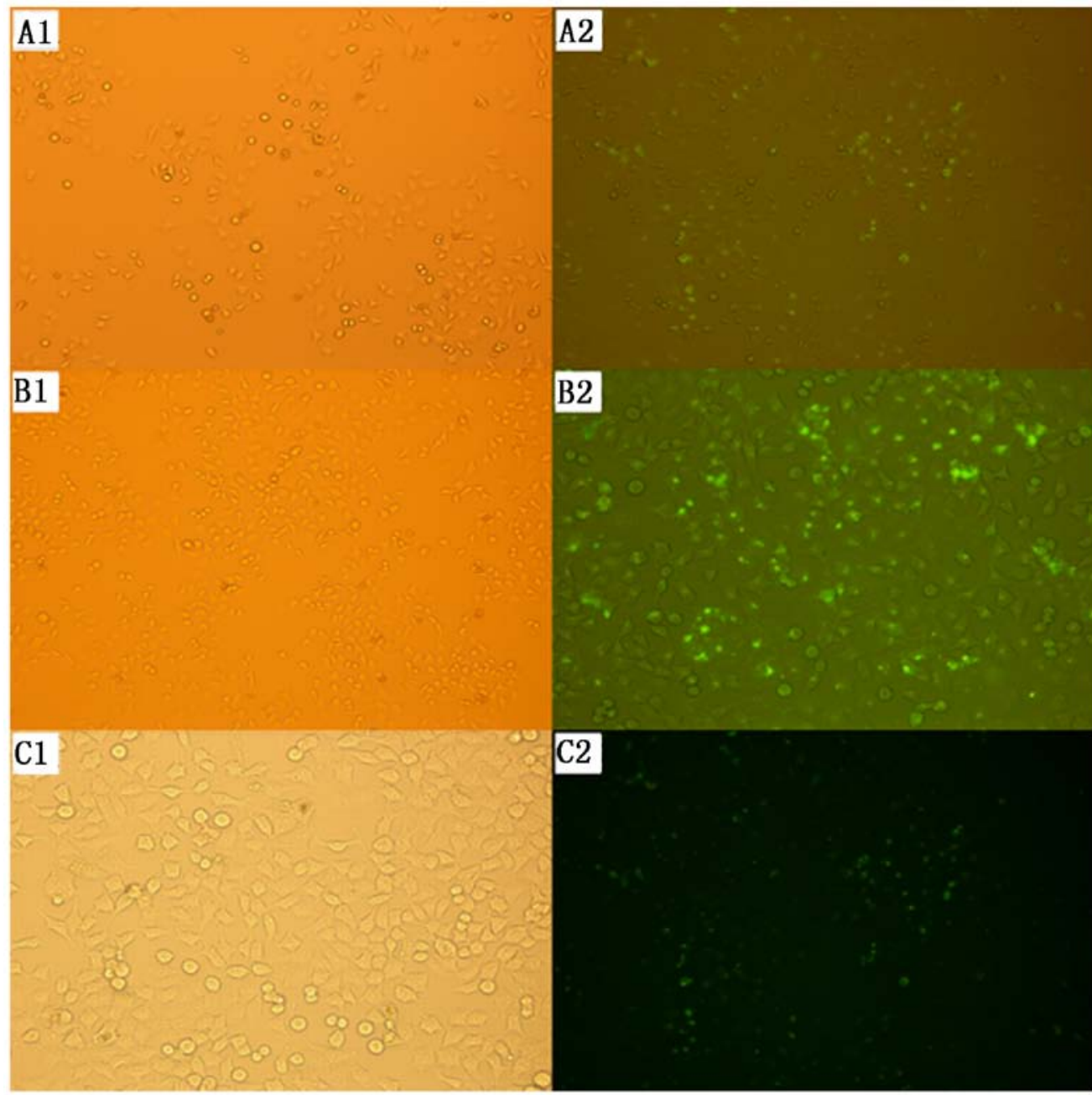

Figure 1. Green fluorescent particles appeared in the SKOV3 cells in all three groups [(A1 and A2) siRNA1 transfected at 24, 48 h; (B1 and B2) siRNA2 transfected at 24, $48 \mathrm{~h} ;(\mathrm{C} 1$ and $\mathrm{C} 2)$ siRNA3 transfected at 24, $48 \mathrm{~h}$, suggesting that siRNA was successfully transfected into the cells.

was specifically expressed in the cytoplasm of endothelial cells in the capillaries of the xenografted tumors. The labeled capillaries presented a brown-yellowish lumen, with either the absence or presence of red blood cells in the lumen. The tumor cells did not express CD34. After podoplanin staining, the endothelial cells in the microlymphatic vessels appeared brown-yellowish, and the microlymphatic vessels exhibited a brown-yellowish-stained lumen. The lymphatic vessels had a thinner wall and a larger lumen compared with the blood vessels, and the lumen of the lymphatic vessels appeared collapsed with no red blood cells. The closed or non-closed linear lumen formed by linking individual brown-yellowishstained endothelial cells or cell clusters was an indicator used to determine the microvessel density (MVD) and lymphatic microvessel density (LMVD) (Fig. 4). The results showed a markedly higher MVD and LMVD in the control groups compared to the intervention group, and these differences were significant (Table III).

Suppression of NFATcl activity results in inhibition of $I L-8$, FGF-2 and PDGF BB mRNA expression in SKOV3 cells. The expression of NFATc1/IL-8/FGF-2/PDGF BB messenger RNA (mRNA) in control groups and siRNA group was assessed by reverse transcriptase polymerase chain reaction. The final gel electrophoresis pictures were analyzed with quantitative one image analysis (Bio-Rad). The OD260/OD280 ratio of total RNA for all samples was in the range of 1.8-2.0, indicating an acceptable purity of the extracted total RNA. After agarose gel electrophoresis of $5 \mu 1$ of total RNA, three bands of $28 \mathrm{~S}, 18 \mathrm{~S}$ and $5.8 \mathrm{~S}$ were clearly present, indicating that the RNA samples were not degraded and could be used for subsequent RT-PCR assays. The results of agarose gel electrophoresis demonstrated that the intensities of the bands corresponding to the reference intracellular $\beta$-actin gene fragments in each group were the same, verifying that the amount of template used for RT-PCR was consistent among the groups. The RT-PCR results, which revealed significant differences in the band intensities of NFATc1, IL-8, FGF-2 and PDGF BB genes among different groups. The intensities of the amplification bands were higher in the blank group and the negative control group and lower in the NFATc1 siRNA intervention group. The grayscale values of NFATc1, IL-8, FGF-2, PDGF BB, and the internal reference $\beta$-actin were obtained using the Quantity One Gel Imaging system and employed to calculate the OD ratios. Compared with the two control groups, the OD ratio in the intervention group showed signifi- 

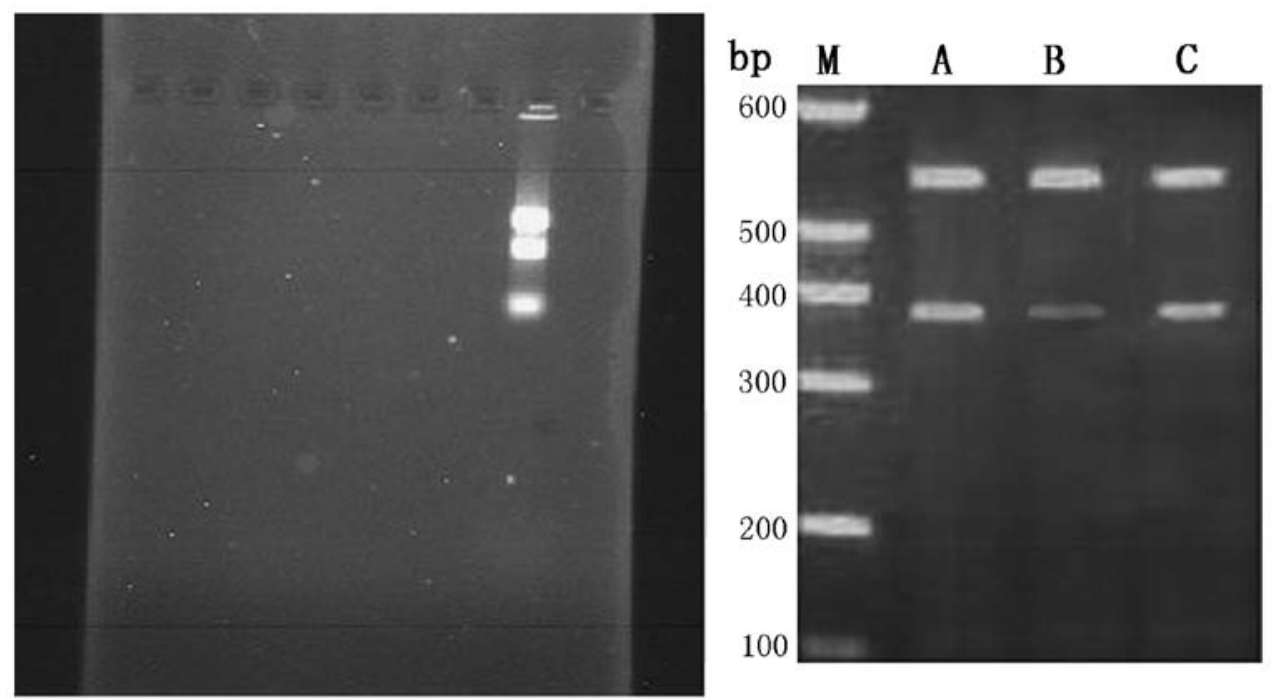

Figure 2. RT-PCR was used to determine the suppression level of NFATc1 gene expression by siRNA in each group at $48 \mathrm{~h}$ post-transfection. Group B presented the highest inhibition rate at $48 \mathrm{~h}$ post-transfection, showing significant differences from the rates of the other two groups $(\mathrm{P}<0.01)$, while no significant difference was observed between group A and C.

Table I. Statistical analysis of NFATc1 mRNA in each transfection group after treated with siRNA $48 \mathrm{~h}$.

\begin{tabular}{lcc}
\hline Group & OD & P-value \\
\hline siRNA1 & $0.532 \pm 0.001$ & P1 \\
siRNA2 & $0.278 \pm 0.001$ & P2 \\
siRNA3 & $0.498 \pm 0.003$ & P3 \\
\hline
\end{tabular}

Transfection group 2, $48 \mathrm{~h}$ compare to each of the other groups $\mathrm{P}<0.01$.

cant differences $(\mathrm{P}<0.05)$, confirming that NFATc1 siRNA can markedly suppress the transcription of the above four genes at the mRNA level (Fig. 5 and Table IV).

Regulation of NFATc1/IL-8/FGF-2/PDGF BB protein by NFATc1 siRNA. The expression of NFATc1 (91 bp)/IL-8 (11 bp)/FGF-2 (15.8 bp)/PDGF BB (24.4 bp) proteins in control groups and siRNA group was assessed by western blot analysis. The final protein stripes size of $\beta$-actin (43 bp) was the same. The expression of these proteins was higher in the control group compared to the siRNA group (Fig. 6 and Table V), the expression level of IL-8/FGF-2/PDGF BB decreased along with the expression of NFATc1, suggesting that there was transcriptional activation of the expression of these proteins in ovarian cancer cells, and NFATc1 siRNA inhibited the protein expression of IL-8/FGF-2/PDGF BB.

\section{Discussion}

It has been reported that NFATc1 can induce the proliferation of pulmonary arterial endothelia cells (23) and enhance the migration and transition of human pulmonary arterial endothelia cells (24). NFATc1 is also a key factor involved in the development of the central vessels during embryo formation. Through synergistic effects with specific ligand cofactors
Table II. Analysis of the transplanted tumor of nude mouse (mean $\pm \mathrm{SD}, \mathrm{n}=18$ ).

\begin{tabular}{lccc}
\hline Treatment & $\begin{array}{c}\text { Weight } \\
(\mathrm{g})\end{array}$ & $\begin{array}{c}\text { Volume } \\
\left(\mathrm{cm}^{3}\right)\end{array}$ & $\begin{array}{c}\text { Inhibitory } \\
\text { rate (\%) }\end{array}$ \\
\hline Blank control & $2.03 \pm 0.35$ & $1.328 \pm 145$ & 0 \\
Negative control & $1.98 \pm 0.78$ & $1.274 \pm 209$ & 0 \\
siRNA & $0.87 \pm 0.32^{\mathrm{a}}$ & $0.512 \pm 087^{\mathrm{a}}$ & $57.08^{\mathrm{a}}$ \\
\hline
\end{tabular}

${ }^{\mathrm{a}} \mathrm{P}<0.05$ vs. blank control and negative control.

[e.g., mitogen-activated protein kinase (MAPK)/extracellular signal-regulated kinase (ERK) kinase I (MEK1)-ERK1/2 and c-Jun N-terminal kinase (JNK)1/2], NFATc1 promotes regulation of the development of embryonic vessels (25). The importance of the VEGF/NFATc1/COX2 signaling pathway implies a pivotal role of NFATc1 in angiogenesis and lymphangiogenesis. However, studies on the effect of NFATc1 in malignant tumors remain scarce, particularly in relation to tumor angiogenesis. Therefore, study on the role of NFATc1 in malignant tumor angiogenesis and the underlying mechanisms is of great significance for the further exploration of related molecular signaling pathways, tumor angiogenesis and lymphangiogenesis and for the identification of effective target genes for treatment.

After establishing a nude mouse model of human ovarian carcinoma xenografts, xenografted tumor tissue was collected and subjected to CD34 staining for microvessel labeling and podoplanin staining for microlymphatic vessel labeling. IHC technology was employed to detect vascular angiogenesis and lymphangiogenesis before and after the NFATc1 siRNA intervention in the xenografted tumor tissue. MVD and LMVD were $12.00 \pm 1.65$ and $10.03 \pm 0.96$, respectively, in the tumor tissue without NFATc1 siRNA intervention, while these values were $5.36 \pm 0.34$ and $4.67 \pm 0.26$ in the tumors subjected to the 


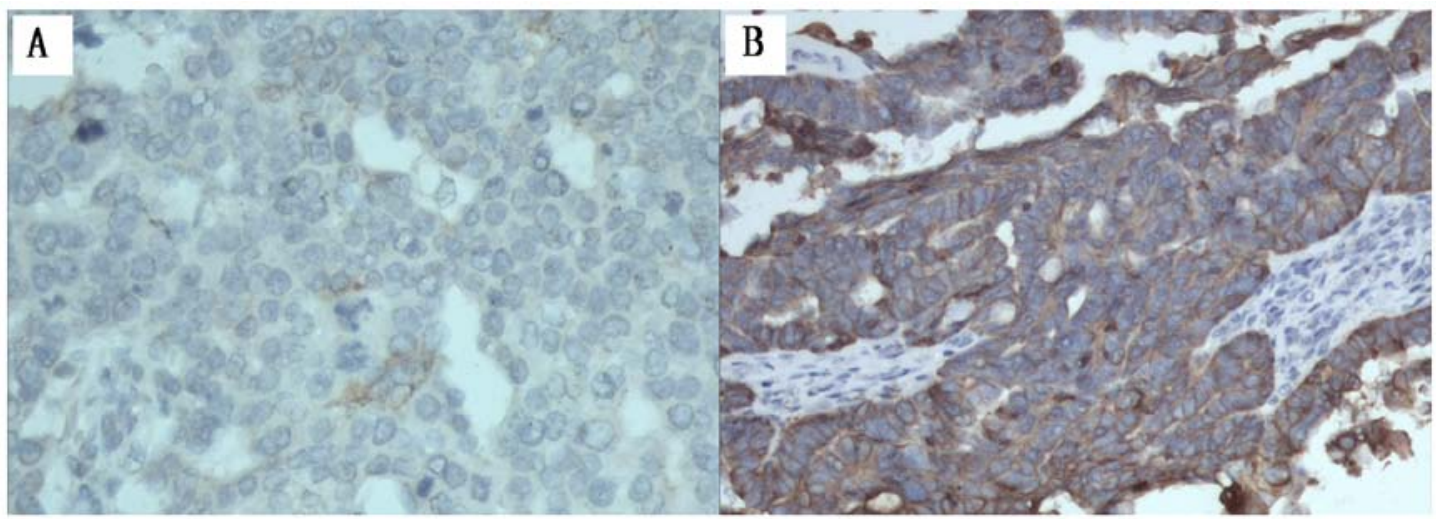

Figure 3. We examined NFATc1 expression in xenograft tumor tissue by immunohistochemical staining. Control group (B) expressed high levels of NFATc1 compared with siRNA group (A).

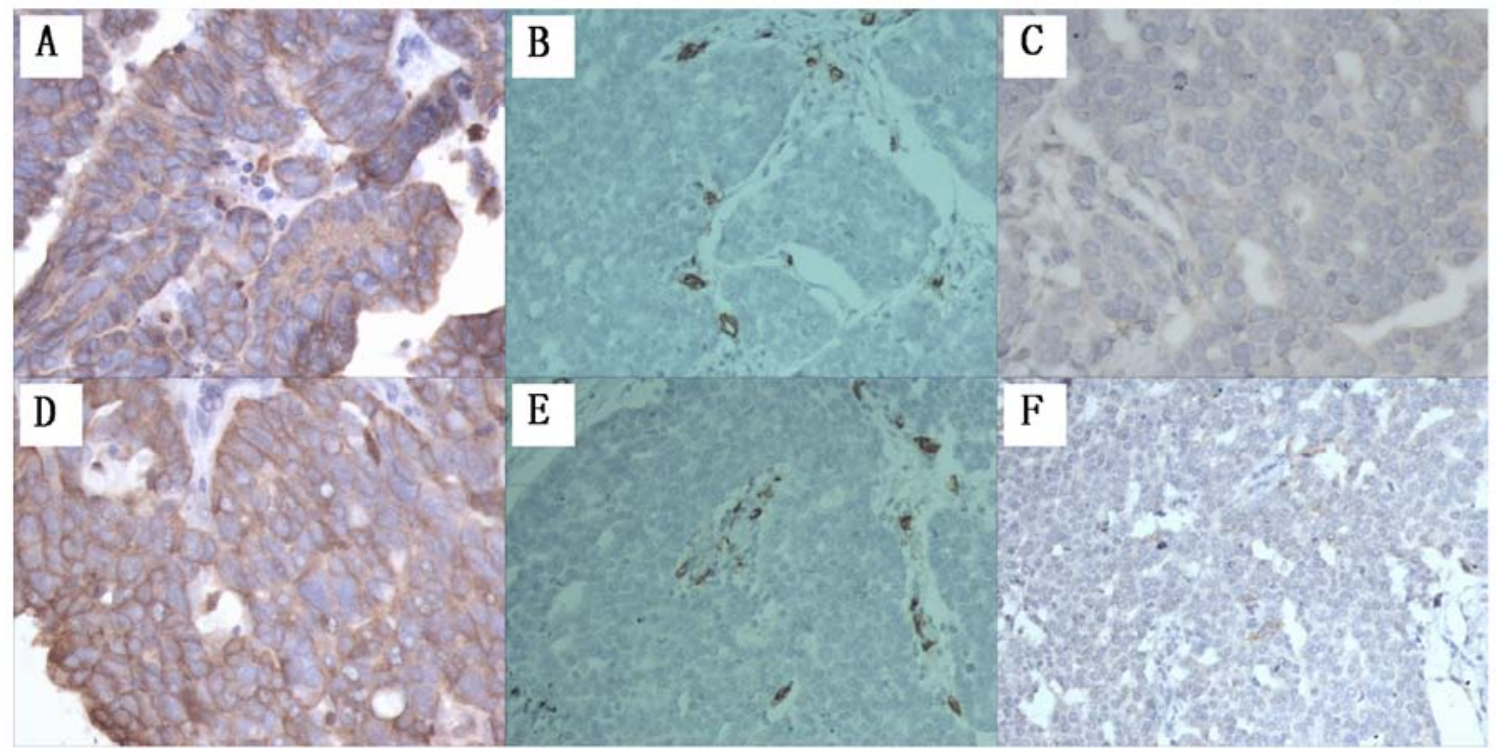

Figure 4. The overall blood vessel density and lymph vessels were decreased statistically in tissues expressing NFATc1 siRNA, as indicated by the reduced number of tissue microvessels stained with CD34 and podoplanin. The brown-yellowish-stained cytokeratin (CK) antigen appeared specifically in the cytoplasm of epithelial cells in all tissue sections of the xenografted tumors (A,D), confirming the epithelial origin of the xenografted tumors. CD34 staining using an anti-CD34 antibody revealed the angiogenesis status in the tumor tissue of each group: CD34 was specifically expressed in the cytoplasm of endothelial cells in the capillaries of the xenografted tumors. The labeled capillaries presented a brown-yellowish lumen, with either the absence or presence of red blood cells in the lumen. The tumor cells did not express CD34 (B: Control; C: siRNA). After podoplanin staining, the endothelial cells in the microlymphatic vessels appeared brown-yellowish, and the microlymphatic vessels had a brown-yellowish-stained lumen (E: Control; F: siRNA). The lymphatic vessels presented a thinner wall and a larger lumen compared with the blood vessels, and the lumen of the lymphatic vessels appeared to be collapsed with no red blood cells. The closed or non-closed linear lumen formed by linking individual brown-yellowish-stained endothelial cells or cell clusters was an indicator used to determine the microvessel density (MVD) and lymphatic microvessel density (LMVD).

Table III. Analysis of IHC, HScore, MVD and LMVD in different groups (mean \pm SD).

\begin{tabular}{|c|c|c|c|c|}
\hline Treatment & Positive rate & HScore & MVD & LMVD \\
\hline Blank control & $100 \%$ & $320(0-356)$ & $12.00 \pm 1.65$ & $10.03 \pm 0.96$ \\
\hline Negative control & $100 \%$ & $336(0-356)$ & $11.47 \pm 0.32$ & $9.95 \pm 1.12$ \\
\hline siRNA & $33.3 \%(10 / 30)^{\mathrm{a}}$ & $35(0-180)^{\mathrm{a}}$ & $5.36 \pm 0.34^{\mathrm{a}}$ & $4.67 \pm 0.26^{\mathrm{a}}$ \\
\hline
\end{tabular}

HScore $=\Sigma \mathrm{pi}(\mathrm{i}+1): \mathrm{i}$ is the intensity of staining, $\mathrm{Pi}$ is the percentage of cells with positive staining/the total number of tested cells, and 1 is the correction factor. ${ }^{a} \mathrm{P}<0.05$ vs. blank control and negative control.

NFATc1 siRNA intervention. Inhibition of NFATc1 expression significantly reduced vascular angiogenesis and lymphan- giogenesis in epithelial ovarian carcinomas, indicating that NFATc1 can promote these two processes in epithelial ovarian 
Table IV. Statistical analysis of NFATc1, IL-8, FGF-2 and PDGF BB mRNA.

\begin{tabular}{lcccc}
\hline Group & NFATc1 & IL-8 & FGF-2 & PDGF BB \\
\hline A & $0.912 \pm 0.001$ & $0.908 \pm 0.003$ & $0.895 \pm 0.005$ & $0.913 \pm 0.005$ \\
B & $0.896 \pm 0.001$ & $0.872 \pm 0.005$ & $0.893 \pm 0.001$ & $0.873 \pm 0.001$ \\
C & $0.265 \pm 0.003$ & $0.325 \pm 0.001$ & $0.296 \pm 0.001$ & $0.218 \pm 0.003$ \\
\hline
\end{tabular}

Comparison of each group of genes: $\mathrm{P} 1, \mathrm{~A}: \mathrm{B}>0.05$; $\mathrm{P} 2, \mathrm{~B}: \mathrm{C}<0.05 ; \mathrm{P} 3, \mathrm{~A}: \mathrm{C}<0.05$.

Table V. Statistical analysis of NFATc1, IL-8, FGF-2 and PDGF BB protein.

\begin{tabular}{lcccc}
\hline Group & NFATc1 & IL-8 & FGF-2 & PDGF BB \\
\hline A & $0.907 \pm 0.001$ & $0.923 \pm 0.001$ & $0.865 \pm 0.005$ & $0.931 \pm 0.003$ \\
B & $0.827 \pm 0.005$ & $0.926 \pm 0.003$ & $0.907 \pm 0.001$ & $0.893 \pm 0.005$ \\
C & $0.295 \pm 0.003$ & $0.376 \pm 0.005$ & $0.396 \pm 0.001$ & $0.318 \pm 0.003$ \\
\hline
\end{tabular}

Comparison of the same protein in each group: P1, A:B $>0.05 ; \mathrm{P} 2, \mathrm{~B}: \mathrm{C}<0.05 ; \mathrm{P} 3$ : A:C $<0.05$.
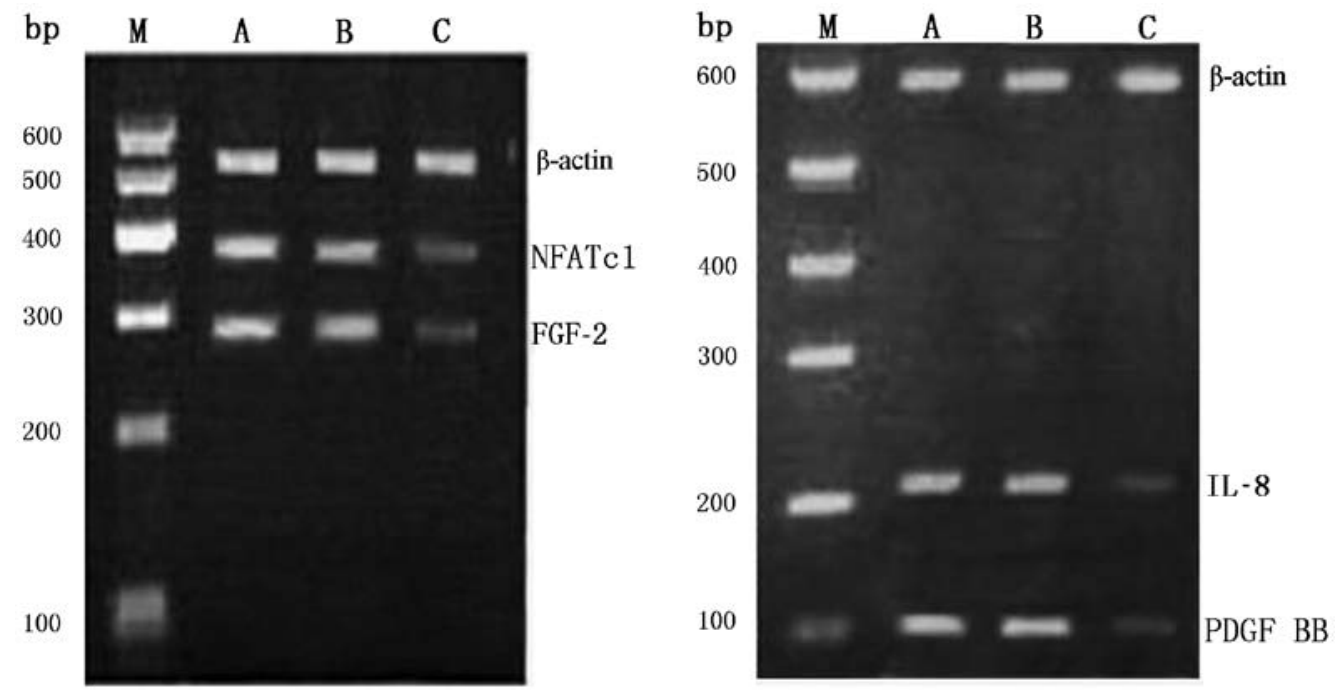

Figure 5. The expression of NFATc1/IL-8/FGF-2/PDGF BB messenger RNA (mRNA) in control groups and siRNA group was assessed by reverse transcriptase polymerase chain reaction. The RT-PCR results revealed significant differences in the band intensities of NFATc1, IL-8, FGF-2 and PDGF BB genes among different groups. The intensities of the amplification bands were higher in the blank group and the negative control group and lower in the NFATc1 siRNA intervention group. The grayscale values of NFATc1, IL-8, FGF-2, PDGF BB, and the internal reference $\beta$-actin were obtained using the Quantity One Gel Imaging system and employed to calculate the OD ratios. Compared with the two control groups, the OD ratio in the intervention group showed significant differences $(\mathrm{P}<0.05)$, confirming that NFATc1 siRNA can markedly suppress the transcription of the above four genes at the mRNA level (Table V).

carcinomas and plays an important role in the angiogenesis of epithelial ovarian carcinomas.

In subsequent experiments, the PCR results showed that the expression levels of the NFATc1, IL-8, FGF-2 and PDGF BB genes were 0.265 $\pm 0.003,0.325 \pm 0.001,0.296 \pm 0.001$ and $0.218 \pm 0.003$, respectively, in the intervention group, which was markedly lower than the levels in the blank control group and the negative control group $(0.912 \pm 0.001,0.908 \pm 0.003$, $0.895 \pm 0.005$ and $0.913 \pm 0.005 ; 0.896 \pm 0.001,0.872 \pm 0.005$, $0.893 \pm 0.001$ and $0.873 \pm 0.001$, respectively), and significant inter-group differences were observed. NFATc1 siRNA markedly suppressed the NFATc1 gene expression level in SKOV3 cells, leading to an accompanying reduction in IL-8,
FGF-2, and PDGF BB expression. The western blot results were consistent with the PCR results. NFATc1 siRNA caused a notable decrease of the NFATc1 protein expression level in SKOV3 cells and an accompanying reduction of IL-8, FGF-2 and PDGF BB protein expression. These differences were all significant. These results suggested that NFATc1 siRNA can inhibit the gene and protein expression of not only NFATc1 but also IL-8, FGF-2 and PDGF BB in SKOV3 epithelial ovarian carcinoma cells.

IL-8, also known as C-X-C motif ligand 8 (CXCL8), is a small soluble protein that belongs to the $\mathrm{CXC}$ chemokine family (26). CXC chemokines can bind to their receptors expressed in endothelial cells and exert extensive effects on 
A

B
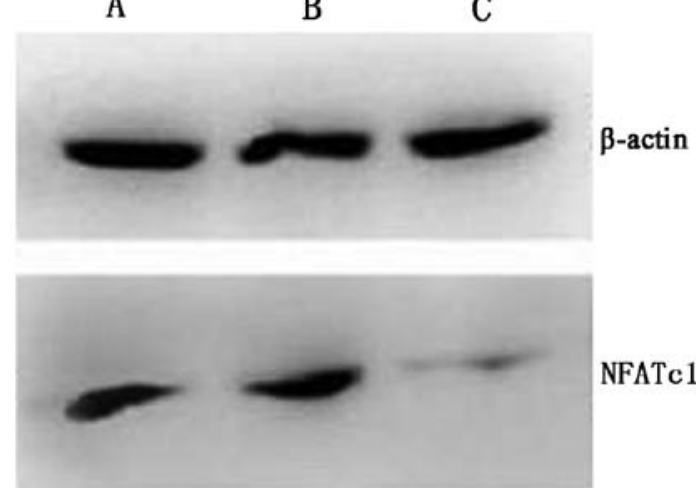

$40 \mathrm{kDa}$
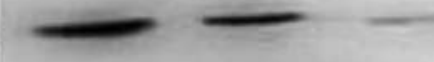

CXCR2

$15.8 \mathrm{kDa}$

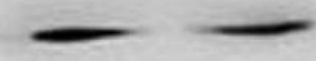

FGF-2

$24.4 \mathrm{kDa}$

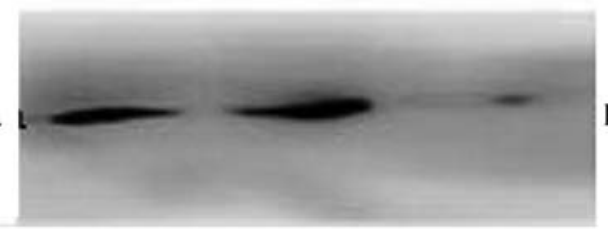

PDGF BB

Figure 6. The expression of NFATc1 (91 bp)/IL-8 (11 bp)/FGF-2 (15.8 bp)/PDGF BB (24.4 bp) proteins in control groups and siRNA group was assessed by western blot analysis. The final protein size of $\beta$-actin (43 bp) was the same. The expression of these proteins was higher in the control group compared to the siRNA group, the expression level of IL-8/ FGF-2/PDGF BB decreased along with the expression of NFATc1, suggesting that there was transcriptional activation of the expression of these proteins in ovarian cancer cells, and NFATc1 siRNA inhibited the protein expression of IL-8/FGF-2/PDGF BB.

angiogenesis through several different mechanisms. The CXC family is characterized by CXC chemokines that can be distinguished based on the expression of a Glu-Leu-Arg (ELR) sequence at the $\mathrm{N} 2$ terminus. ELR exerts potent physiological effects on angiogenesis. In contrast to the $\mathrm{ELR}^{+} \mathrm{CXC}$ chemokines, which promote angiogenesis, all ELR-free chemokines are essentially anti-angiogenic. One of the representative $\mathrm{ELR}^{+}$ chemokine is CXCL8/IL8, which was discovered in 1992 and is a potent inducer of and factor underlying angiogenesis. The key role of $\mathrm{ELR}^{+} \mathrm{CXC}$ chemokines in angiogenesis determines their fundamental position in the abnormal regulation of angiogenesis during the development of malignant tumors. This viewpoint is supported by a great number of studies (27-30). In addition, IL-8 can act on various signaling pathways, such as the phosphatidylinositol 3-kinase (PI3K)/protein kinase B (AKT), nuclear factor (NF)- $\mathrm{B}$, MAPK and signal transducer and activator of transcription-3 (Stat-3) pathways, to facilitate angiogenesis, strongly demonstrating its key role in the occurrence and development of malignant tumors.
It has been shown that the formation and shaping of lymphatic vessels occur actively in malignant tumors, and tumor-induced lymphangiogenesis is initiated by lymphangiogenic factors, among which FGF-2 and PDGF BB are two important factors $(31,32)$. Previous studies have identified FGF-2 as a pro-angiogenesis growth factor, potent mitogen and chemoattractant (33). Studies conducted in recent years confirmed the close relationship of FGF-2 with tumor lymphangiogenesis and lymphatic metastasis, and its important role in tumor lymphangiogenesis is supported by the observation that a low expression level of FGF-2 cannot induce angiogenesis but can specifically induce tumor lymphangiogenesis (34). Platelet-derived growth factor BB is a member of the PDGF family. It was revealed that PDGF BB can stimulate tumor lymphangiogenesis and lymphatic metastasis, thus serving as an independent lymphangiogenic factor. One major function of PDGF BB is to stabilize the vascular network; however, recent studies revealed that PDGF can directly stimulate lymphangiogenesis and lymphatic metastasis (35) and enhance lymphangiogenesis independent of VEGFR-3 to promote tumor growth and metastasis. Specifically targeting PDGF $\mathrm{BB}$ is a new strategy for the inhibition of lymphangiogenesis. IHC experiments demonstrated that FGF-2 and PDGF BB promoted lymphangiogenesis in inflammatory bowel disease. Using an NFATc1 siRNA intervention technique, this study also showed that NFATc1 could affect FGF-2 and PDGF BB, as demonstrated by the finding that the expression levels of FGF-2 and PDGF BB decreased with a decrease in NFATc1 expression, indicating that the effect of NFATc1 on lymphangiogenesis in epithelial ovarian carcinoma may be mediated by FGF-2 and PDGF BB regulation.

In addition, COX-2 activation during the promotion of angiogenesis and tumor invasion can increase the expression levels of PDGF and bFGF (36). Moreover, PDGF activation by VEGF relies on COX2 overexpression (37).

In addition, COX-2 activation during the promotion of angiogenesis and tumor invasion can increase the expression levels of PDGF and bFGF (33). Moreover, PDGF activation by VEGF relies on COX2 overexpression (34). In endothelial cells, stimulation of FGF-2 can lead to hyperresponsiveness of PDGF BB, which in turn results in augmentation of receptor expression in the wall cells of lymphatic vessels and generates positive feedback to the FGF-2 signal. Ultimately, the uncoordinated interactions between these two proteins lead to unregulated formation and shaping of primitive lymphatic vessels in the tumor microenvironment, thus promoting tumor growth and metastasis.

As discussed above, based on the knowledge on the VEGF/NFATc1/COX2 signaling pathway and our results, we can draw the possible conclusion that NFATc1 plays a critical role in angiogenesis in epithelial ovarian carcinoma. When activated by VEGF-A or VEGF-C, NFATc1 acts on downstream IL-8 to promote angiogenesis through regulating the expression of $\mathrm{ELR}^{+} \mathrm{CXC}$ chemokines. Moreover, activated NFATc1 can affect the expression of FGF-2 and PDGF BB through downstream $\mathrm{COX} 2$, thus regulating tumor vascular angiogenesis and lymphangiogenesis. FGF-2 and PDGF BB can form a positive feedback loop through which the two proteins interact with and affect each other, thus jointly accelerating the occurrence and metastasis of tumors by promoting tumor angiogenesis. The 
above signaling pathway may be a promising target for the treatment of epithelial ovarian carcinoma.

\section{Acknowledgements}

This study was supported by the National Science Foundation of China (no. 81402126).

\section{References}

1. Naito T, Tanaka H, Naoe Y and Taniuchi I: Transcriptional control of T-cell development. Int Immunol 23: 661-668, 2011.

2. Chen L, Glover JN, Hogan PG, Rao A and Harrison SC: Structure of the DNA-binding domains from NFAT, Fos and Jun bound specifically to DNA. Nature 392: 42-48, 1998.

3. Jain J, McCaffrey PG, Valge-Archer VE and Rao A: Nuclear factor of activated $\mathrm{T}$ cells contains Fos and Jun. Nature 356 801-804, 1992

4. Bengsch B and Wherry EJ: The importance of cooperation: partnerless NFAT induces T cell exhaustion. Immunity 42: 203-205, 2015.

5. Shaw JP, Utz PJ, Durand DB, Toole JJ, Emmel EA and Crabtree GR: Identification of a putative regulator of early $\mathrm{T}$ cell activation genes. Science 241: 202-205, 1988.

6. Bai S and Kerppola TK: Opposing roles of FoxP1 and Nfat3 in transcriptional control of cardiomyocyte hypertrophy. Mol Cell Biol 31: 3068-3080, 2011.

7. Hanahan D and Weinberg RA: The hallmarks of cancer. Cell 100: 57-70, 2000.

8. Chen NM, Singh G, Koenig A, Liou GY, Storz P, Zhang JS, Regul L, Nagarajan S, Kühnemuth B, Johnsen SA, et al: NFATc1 links EGFR signaling to induction of Sox9 transcription and acinar-ductal transdifferentiation in the pancreas. Gastroenterology 148: 1024-1034.e9, 2015.

9. Hashizume M, Hayakawa N and Mihara M: IL-6 trans-signalling directly induces RANKL on fibroblast-like synovial cells and is involved in RANKL induction by TNF-alpha and IL-17. Rheumatology (Oxford) 47: 1635-1640, 2008.

10. Wang S, Kang X, Cao S, Cheng H, Wang D and Geng J: Calcineurin/NFATc1 pathway contributes to cell proliferation in hepatocellular carcinoma. Dig Dis Sci 57: 3184-3188, 2012.

11. Beals CR, Sheridan CM, Turck CW, Gardner P and Crabtree GR: Nuclear export of NF-ATc enhanced by glycogen synthase kinase-3. Science 275: 1930-1934, 1997.

12. Köenig A, Linhart T, Schlengemann K, Reutlinger K, Wegele J, Adler G, Singh G, Hofmann L, Kunsch S, Büch T, et al: NFAT-induced histone acetylation relay switch promotes c-Myc-dependent growth in pancreatic cancer cells. Gastroenterology 138: 1189-1199.e1-2, 2010.

13. Neal JW and Clipstone NA: A constitutively active NFATc1 mutant induces a transformed phenotype in 3T3-L1 fibroblasts. J Biol Chem 278: 17246-17254, 2003.

14. Zhang SD, McCrudden CM, Meng C, Lin Y and Kwok HF: The significance of combining VEGFA, FLT1, and KDR expressions in colon cancer patient prognosis and predicting response to bevacizumab. Onco Targets Ther 8: 835-843, 2015.

15. Wu B, Baldwin HS and Zhou B: Nfatcl directs the endocardial progenitor cells to make heart valve primordium. Trends Cardiovasc Med 23: 294-300, 2013.

16. Wu B, Wang Y, Lui W, Langworthy M, Tompkins KL, Hatzopoulos AK, Baldwin HS and Zhou B: Nfatcl coordinates valve endocardial cell lineage development required for heart valve formation. Circ Res 109: 183-192, 2011.

17. Adinolfi E, Raffaghello L, Giuliani AL, Cavazzini L, Capece M, Chiozzi P, Bianchi G, Kroemer G, Pistoia V and Di Virgilio F: Expression of P2X7 receptor increases in vivo tumor growth. Cancer Res 72: 2957-2969, 2012.

18. Shoemaker LD, Fuentes LF, Santiago SM, Allen BM, Cook DJ, Steinberg GK and Chang SD: Human brain arteriovenous malformations express lymphatic-associated genes. Ann Clin Transl Neurol 1: 982-995, 2014.
19. Chen HM, Tsai $\mathrm{CH}$ and Hung WC: Foretinib inhibits angiogenesis, lymphangiogenesis and tumor growth of pancreatic cancer in vivo by decreasing VEGFR-2/3 and TIE-2 signaling. Oncotarget 6: 14940-14952, 2015

20. Kähärä J and Lähdesmäki H: Evaluating a linear k-mer model for protein-DNA interactions using high-throughput SELEX data. BMC Bioinformatics 14 (Suppl 10): S2, 2013.

21. Kulkarni RM, Greenberg JM and Akeson AL: NFATc1 regulates lymphatic endothelial development. Mech Dev 126: 350-365, 2009.

22. Norrmén C, Ivanov KI, Cheng J, Zangger N, Delorenzi M, Jaquet M, Miura N, Puolakkainen P, Horsley V, Hu J, et al: FOXC2 controls formation and maturation of lymphatic collecting vessels through cooperation with NFATc1. J Cell Biol 185: 439-457, 2009.

23. Lee JH, Bhang DH, Beede A, Huang TL, Stripp BR, Bloch KD, Wagers AJ, Tseng YH, Ryeom S and Kim CF: Lung stem cell differentiation in mice directed by endothelial cells via a BMP4-NFATc1-thrombospondin-1 axis. Cell 156: 440-455, 2014.

24. Jang GH, Park IS, Yang JH, Bischoff J and Lee YM: Differential function of genes regulated by VEGF-NFATc1 signaling pathway in migration of pulmonary valve endothelial cells. FEBS Lett 584: 141-146, 2010.

25. Combs MD and Yutzey KE: VEGF and RANKL regulation of NFATc1 in heart valve development. Circ Res 105: 565-574, 2009.

26. Li WH, Qiu Y, Zhang HQ, Tian XX and Fang WG: P2Y2 Receptor and EGFR cooperate to promote prostate cancer cell invasion via ERK1/2 pathway. PLoS One 10: e0133165, 2015.

27. Moldobaeva A, Baek A, Eldridge L and Wagner EM: Differential activity of pro-angiogenic CXC chemokines. Microvasc Res 80: $18-22,2010$

28. Joimel U, Gest C, Soria J, Pritchard LL, Alexandre J, Laurent M, Blot E, Cazin L, Vannier JP, Varin R, et al: Stimulation of angiogenesis resulting from cooperation between macrophages and MDA-MB-231 breast cancer cells: Proposed molecular mechanism and effect of tetrathiomolybdate. BMC Cancer 10: 375,2010

29. Sarmiento J, Shumate C, Suetomi K, Ravindran A, Villegas L, Rajarathnam K and Navarro J: Diverging mechanisms of activation of chemokine receptors revealed by novel chemokine agonists. PLoS One 6: e27967, 2011.

30. Rabquer BJ, Tsou PS, Hou Y, Thirunavukkarasu E, Haines GK III, Impens AJ, Phillips K, Kahaleh B, Seibold JR and Koch AE: Dysregulated expression of MIG/CXCL9, IP-10/CXCL10 and CXCL16 and their receptors in systemic sclerosis. Arthritis Res Ther 13: R18, 2011.

31. Caoa R, Jia H, Fenga N, et al: Collaborative interplay between FGF-2 and VEGF-C promotes lymphangiogenesis and metastasis. PNAS 109: 15894-15899, 2012.

32. Cao R1, Björndahl MA, Religa P, et al: PDGF-BB induces intratumoral lymphangiogenesis and promotes lymphatic metastasis. Cancer Cell 6: 333-345, 2004.

33. Andrés G1, Leali D, Mitola S, et al: A pro-inflammatory signature mediates FGF2-induced angiogenesis. Molecular Medicine 13: 2083-2108, 2009.

34. Chang LK, Garcia-Cardeña G, Farnebo F, Fannon M, Chen EJ, Butterfield C, Moses MA, Mulligan RC, Folkman J, Kaipainen A: Dose-dependent response of FGF-2 for lymhangiongenesis. Proc Natl Acad Sci USA 101: 11658-11663, 2004.

35. Schoppmann SF, Alidzanovic L, Schultheis A, Perkmann T, Brostjan C and Birner P: Thrombocytes correlate with lymphangiogenesis in human esophageal cancer and mediate growth of lymphatic endothelial cells in vitro. PLoS One 8: e66941, 2013.

36. Tung HC, Lee FY, Wang SS, Tsai MH, Lee JY, Huo TI, Huang HC, Chuang CL, Lin HC and Lee SD: The beneficial effects of P2X7 antagonism in rats with bile duct ligation-induced cirrhosis. PLoS One 10: e0124654, 2015.

37. Zhao Y, Wang Y, Wang Q, Liu Z, Liu Q and Deng X: Hepatic stellate cells produce vascular endothelial growth factor via phospho-p44/42 mitogen-activated protein kinase/cyclooxygenase-2 pathway. Mol Cell Biochem 359: 217-223, 2012. 